\title{
Non-invasive indirect monitoring of intra-abdominal pressure using microwave reflectometry: system design and proof-of-concept clinical trial
}

\author{
Marcelo David ${ }^{1}$ (D ) Aviad Raviv ${ }^{1} \cdot$ Ariel Guttel $^{1} \cdot$ Valentina García Reyes $^{2} \cdot$ Franco Simini $^{3} \cdot$ Francisco Pracca $^{2}$
}

Received: 22 June 2020 / Accepted: 5 October 2020 / Published online: 14 October 2020

C) Springer Nature B.V. 2020

\begin{abstract}
Monitoring intra-abdominal pressure (IAP) has become a standard in intensive care units. Correlation between the abdominal wall's thickness (AWTh) and IAP has been reported previously. The abdominal wall can be modeled as a compound of parallel dielectric slabs; changes in their width have a direct effect on the reflection coefficient of the abdominal wall at microwave frequencies. This work describes the design of a reflectometry system and its proof-of-concept trial on five patients during laparoscopic surgery. The system complies with IEEE Std. C95.1-2005 concerning exposure of humans to microwave electromagnetic fields in controlled environments. The results putatively show an inverse correlation between IAP and the reflection coefficient, and a strong dependence on the body mass index. A better understanding of the dynamics in the changes of the AWTh (during intra-abdominal hypertension) will allow further development of a microwave-based technique for the continuous non-invasive indirect monitoring of IAP in critical patients.
\end{abstract}

Keywords Intra-abdominal pressure $\cdot$ Microwave reflectometry $\cdot$ Non-invasive

\section{Introduction}

Intra-abdominal pressure (IAP) is the steady-state pressure concealed within the abdominal cavity [1]. The monitoring of IAP has become a standard in intensive care units (ICUs) as intra-abdominal hypertension (IAH) is directly associated with increased morbidity and mortality [2]. This relies on the fact that IAH (i.e. IAP over $12 \mathrm{mmHg}$ ) entails a negative impact in abdominal organ perfusion which could lead to organ dysfunction [2]. The most feared consequence of IAH, known as abdominal compartment syndrome (ACS), is defined as a sustained IAP $>20 \mathrm{mmHg}$, associated with severe organ dysfunction [2-5]. By monitoring IAP, our ultimate goal is to guarantee an adequate organ blood flow

Marcelo David

marcelod@jct.ac.il

1 Department of Electrical Engineering, Jerusalem College of Technology - Lev Academic Center, Jerusalem, Israel

2 Department of Intensive Medicine, Hospital de Clínicas, Universidad de la República, Montevideo, Uruguay

3 Núcleo de Ingeniería Biomédica, Universidad de la República, Montevideo, Uruguay and, consequently, to reduce the risk of organ dysfunction, employing corresponding treatments [6]. Since IAH affects about $54-59 \%$ of critical patients [1, 7], monitoring IAP is usually indicated in ICUs $[2,8]$.

Diverse techniques are used in intensive care for monitoring IAP $[9,10]$. Continuous monitoring of IAP has been researched by several groups in the past years $[2,3$, 11-13], but no feasible or easy-to-use non-invasive method has reached clinical use yet [3]. In 2017, David et al. [14] proposed a generic numerical electromagnetic model of the abdominal wall as for correlating abdominal wall's thickness (AWTh) to microwave reflectometry. In 2018, David et al. verified the theory by preliminary results on porcine models [15].

This work presents the design and a proof-of-concept clinical trial of AbdoRF: an easy-to-use, portable, microwave reflectometry system (in the frequency range between 3.90 and $4.45 \mathrm{GHz}$ ), which complies with IEEE C95 standards regarding exposure of humans to non-ionizing electromagnetic radiation in controlled environments. 


\section{Materials and methods}

\subsection{Patients}

The target population were patients hospitalized in the Hospital de Clínicas-Universidad de la República, admitted by the Emergency Department for laparoscopy surgery due to acute abdominal pathologies.

Inclusion criteria:

- Adults (at least 18 years old) from the target population.

- Ability to accept or decline signing the informed consent.

Exclusion criteria:

- Pregnant.

- Vesical or pelvis trauma.

- Obstructive uropathy.

- Urosepsis.

\subsection{System design and description}

The abdominal wall mainly consists of five soft tissue layers as described in [16-19]:

1. Skin and subcutaneous tissue

2. Fascia

a. Camper's fascia (fat)

b. Scarpa's fascia (fibrous)

3. Muscle

4. Fascia transversalis

5. Peritoneum

A detailed description of the electromagnetic (dielectric) model of the abdominal wall is presented in [14, 15]. For simplicity, the abdominal wall can be considered a compressible non-homogeneous anisotropic dielectric slab [20-22], which will be compressed as IAP increases and will, therefore, change its thickness. Previously published numerical analysis [14] suggests that such changes in the AWTh, correlate well with the reflection coefficient of the abdominal wall (called $\mathrm{S}_{11}$-input port reflection coefficient). $\mathrm{S}_{11}$ is a ratio between the reflected power $\left(P_{\text {reflected }}\right)$ and transmitted power $\left(P_{\text {transmitted }}\right)$ at the antenna, usually measured in $\mathrm{dB}$ (Eq. (1)) [14].

$S_{11}=10 \cdot \log \left(\frac{P_{\text {reflected }}}{P_{\text {transmitted }}}\right)[\mathrm{dB}]$
AbdoRF is a portable, relatively low-cost microwave reflectometry system, designed to evaluate the reflection coefficient of a patch antenna non-invasively attached to the abdominal wall.

The hardware consists of the following parts as shown in Fig. 1 (design files of the system are available upon reasonable request to the corresponding author):

1. A generic microcontroller that commands and links between the hardware devices.

2. A Voltage Controlled Oscillator (VCO) for radiofrequency wave generation in the range between 3.90 and 4.45 GHz.

3. A wideband flexible patch antenna.

4. A bidirectional RMS detector measures the power of the transmitted and reflected wave through the antenna.

5. A digital-to-analog converter and amplifiers to control the VCO input.

6. Analog-to-digital converters for sampling instantaneous RMS power values measured by (4), used for calculating $\mathrm{S}_{11}$.

\subsection{IEEE Std. C95.1-2005: human exposure to EM fields in controlled environments}

IEEE C95.1 is an approved standard for safety levels concerning human exposure to electric, magnetic and electromagnetic fields. It states that, for electromagnetic fields within the frequency range between 2 and $5 \mathrm{GHz}$, the maximum RMS power density allowed for exposure in controlled environments (such as clinical use) is:

$\Phi_{\max }=10 \frac{\mathrm{W}}{\mathrm{m}^{2}}$

In the clinical trial, the antenna FXP100.07.0100A (Taoglas Ltd.) was used. Given that (according to the antenna's datasheet) the radiation area of the antenna is $A=744 \mathrm{~mm}^{2}$ (see Fig. 2), the maximum power driven to the antenna (in the ideal case of complete transmission from the antenna to the abdominal wall) is:

$P_{\text {max }}=\Phi_{\text {max }} \cdot A=10 \frac{\mathrm{W}}{\mathrm{m}^{2}} \cdot 744 \mathrm{~mm}^{2}=7.44 \mathrm{~mW} \approx 8.7 \mathrm{dBm}$

The VCO used in our system, HMC391 (Analog Devices), supplies a power of only $5 \mathrm{dBm}$; thus, the system complies with the IEEE C95.1 standard.

\subsection{Clinical trial setup}

This proof-of-concept clinical trial is a prospective observational study in which we randomly enrolled five patients 


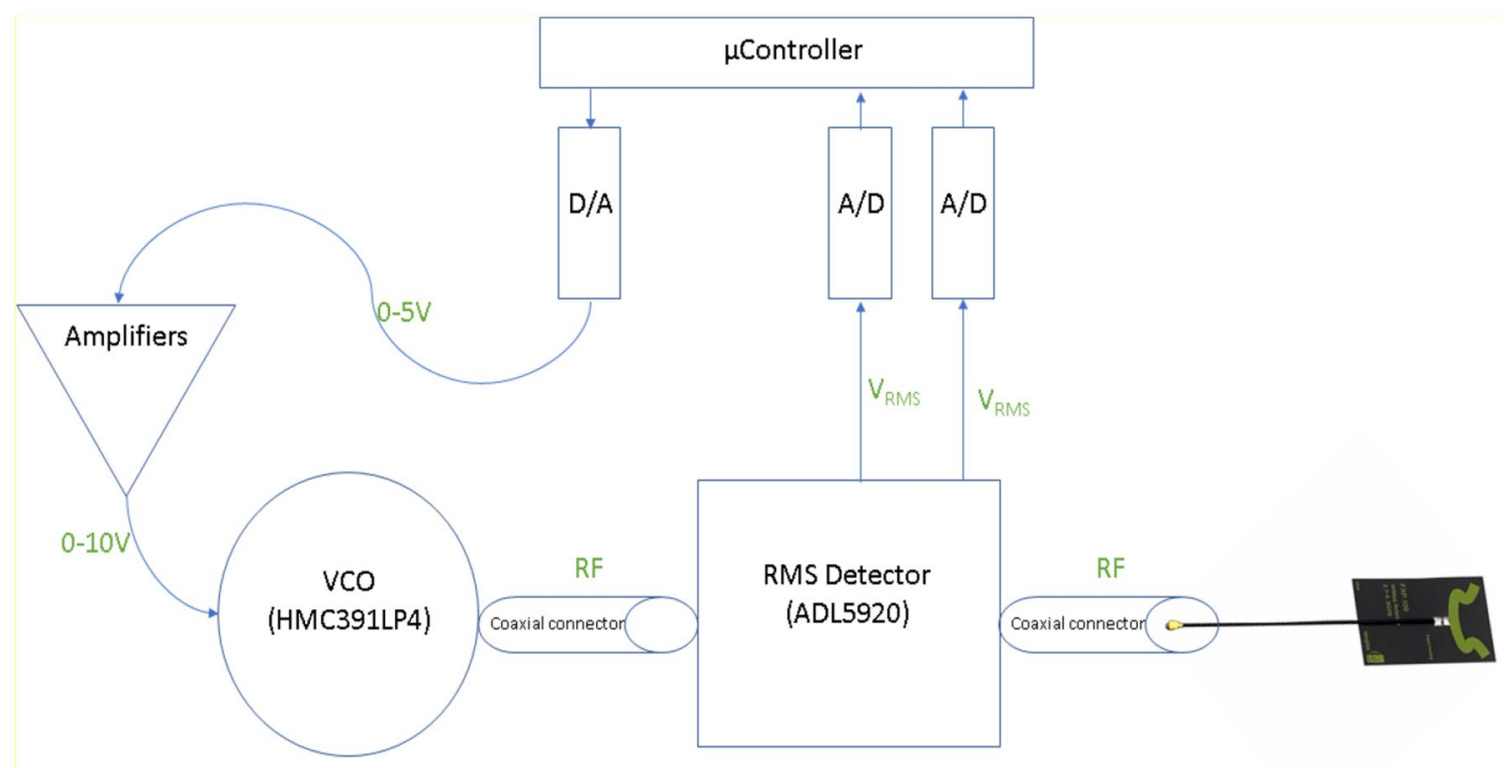

(a)

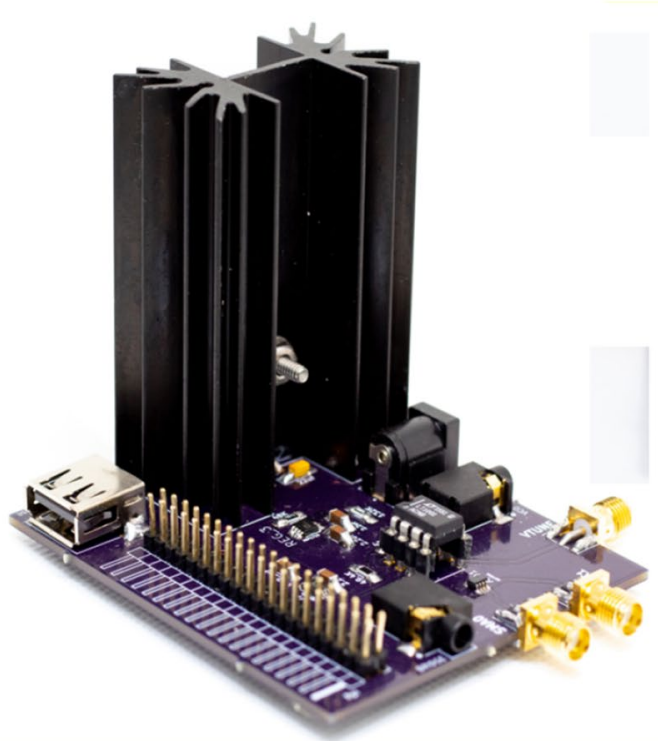

(b)

Fig. 1 AbdoRF a system schematic diagram $\mathbf{b}$ picture of the device

undergoing laparoscopic surgery (see Sects. 2.1 and 2.2). Before anesthetic induction, premedication by $1 \mu \mathrm{g} / \mathrm{kg}$ of fentanyl was supplied. Balanced general anesthesia was performed at inhalation on mechanical ventilator support with orotracheal intubation. Anesthetic induction was intravenous with $2-4 \mathrm{mg} / \mathrm{kg}$ of Propofol, and muscle relaxation with $0.6 \mathrm{mg} / \mathrm{kg}$ of Atracurium. After orotracheal intubation, anesthetic maintenance was performed with 0.5 MAC inhalation isofluorane and remifentanil in the range of $0.25-0.50 \mu \mathrm{g} / \mathrm{kg} /$ min. Muscle relaxation was maintained based on Atracurium $0.3 \mathrm{mg} / \mathrm{kg}$ when required. During mechanical ventilation, the volume-controlled mode was used, with a tidal volume of 6-8 $\mathrm{ml} / \mathrm{kg}$, PEEP in the range of $5-10 \mathrm{cmH}_{2} \mathrm{O}$, adjusting the respiratory rate according to exhaled- $\mathrm{CO}_{2}$ values.

Patients were in supine position; a trocar was inserted across the abdominal wall, reaching the intraperitoneal abdominal cavity for the laparoscopic pneumoperitoneum. Through the trocar, the abdominal cavity was inflated using $\mathrm{CO}_{2}$ (influx of 0-30 $\mathrm{l} / \mathrm{min}$ ) to different pressures as needed for the surgical procedure. As recommended by WSACS (The Abdominal Compartment Society), the induced IAP was kept constant for about $60 \mathrm{~s}$ before performing the 


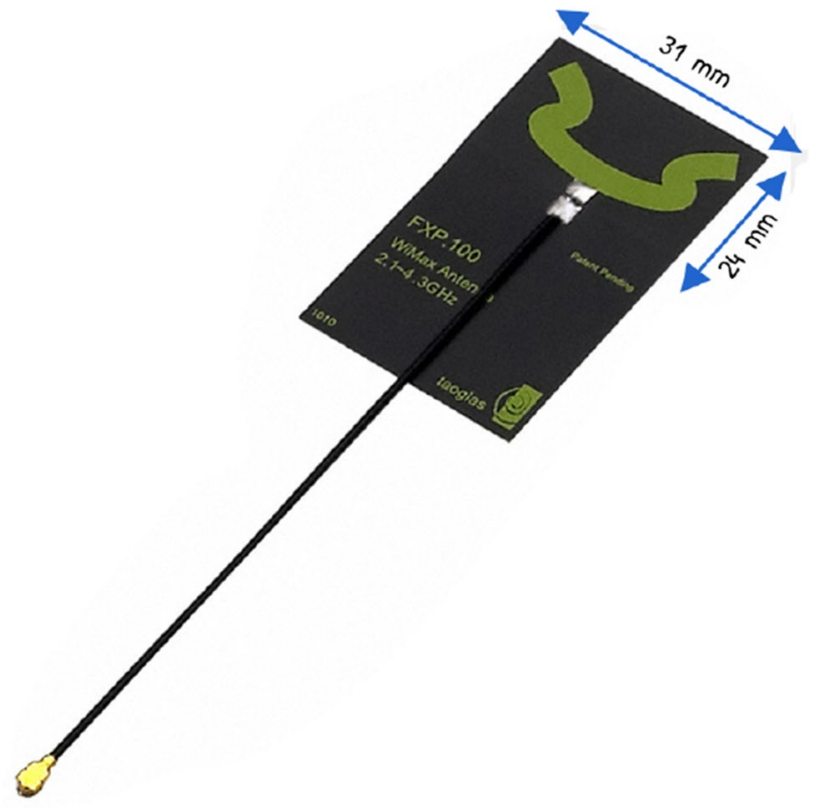

Fig. 2 Antenna FXP100.07.0100A (Taoglas Ltd.). Radiation area $24 \mathrm{~mm} \times 31 \mathrm{~mm}$

measurements $[2,9,10]$, the measurements were performed at the end of expiration. Each reflectometry measurement was performed five times for each IAP. Due to logistical constraints during the surgery, the antenna was placed over the left-inferior quarter of the abdominal wall (except Patient E-see Sect. 3.1), next to the linea alba as shown in Fig. 3. Note that the front-side of the antenna (radiation area) is facing the abdomen, thus its back-side is seen in the picture.

\section{Results}

\subsection{Patients}

Five patients were studied in this clinical trial, as presented in Table 1. Patients A-D underwent cholecystectomy, while Patient $\mathrm{E}$ underwent an appendectomy. In the case of Patient
Table 1 List of patients in the clinical trial and their relevant variables

\begin{tabular}{llllll}
\hline Patient & BMI & Height $[\mathrm{m}]$ & Age & Gender & Diagnosis \\
\hline A & 25.71 & 1.65 & 60 & F & Cholecystectomy \\
B & 17.58 & 1.60 & 60 & F & Cholecystectomy \\
C & 38.05 & 1.58 & 38 & F & Cholecystectomy \\
D & 33.87 & 1.63 & 34 & F & Cholecystectomy \\
E & 23.94 & 1.71 & 21 & M & Appendectomy \\
\hline
\end{tabular}

$\mathrm{E}$, the antenna was placed on the left-superior quarter of the abdominal wall (under the diaphragm).

Since the five patients are approximately the same height, body mass index (BMI-Eq. (4)) can be directly related to the accumulation of fat tissue in the abdominal wall [4]. This, in turn, has an impact on the compressibility of the abdominal wall [4] and its overall wave impedance (and thus the reflection coefficient S11) [14, 15].

$B M I=\frac{\text { weight }[\mathrm{kg}]}{(\text { height }[\mathrm{m}])^{2}}$.

\subsection{Reflectometry results}

Each reflectometry measurement was performed five times for each IAP induced by air insufflation during the laparoscopic procedure. The measurements were averaged and the standard deviation was calculated. Reproducibility of these results depends on a well-coupling of the antenna and the abdominal wall; special care is to be taken to ensure that the whole antenna is touching the abdomen without any separation.

Figure 4 presents absolute values of reflection coefficient $S_{11}$ vs frequency at different IAP for patient A (similar behavior is observed for all patients - raw data are available upon request to the corresponding author). In all patients, maximum sensitivity to changes in IAP was found at a critical frequency of about $4.41 \mathrm{GHz}$ (see Table 2).
Fig. 3 Antenna over the abdominal wall while the patient is in supine position: a schematic sagittal cut; b Patient A-the antenna is placed over the left-inferior quarter of the abdominal wall

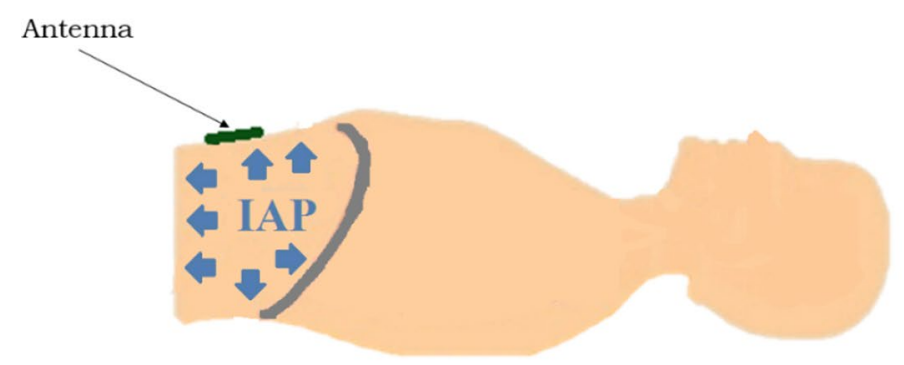

(a)

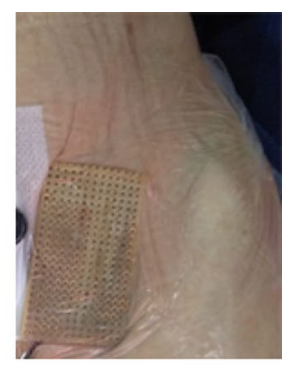

(b) 


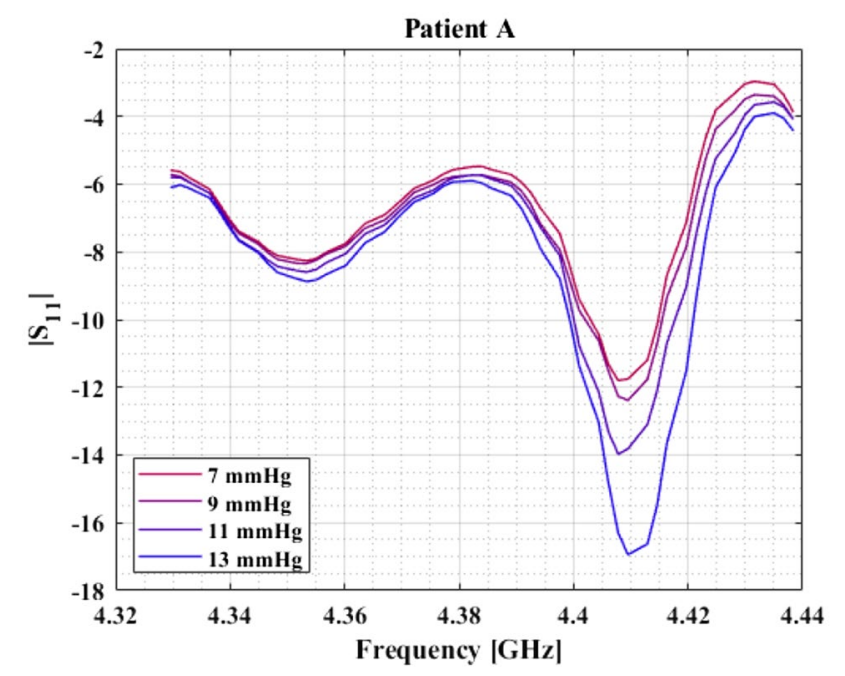

Fig. 4 Absolute values of reflection coefficient in $\mathrm{dB}$ vs. frequency at different IAP for patient A. Similar behavior is observed in every patient

Table 2 Critical frequency (maximum sensitivity)

\begin{tabular}{ll}
\hline Patient & Critical frequency \\
\hline A & $4.408 \mathrm{GHz}$ \\
B & $4.413 \mathrm{GHz}$ \\
C & $4.410 \mathrm{GHz}$ \\
D & $4.415 \mathrm{GHz}$ \\
E & $4.415 \mathrm{GHz}$ \\
\hline
\end{tabular}

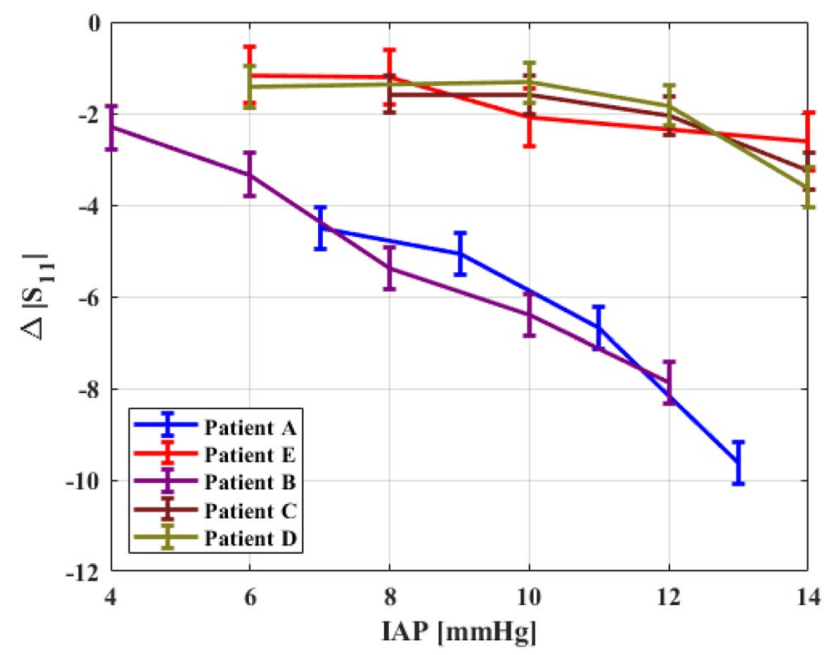

Fig. 5 Relative changes in reflection coefficient (in $\mathrm{dB}$ ) at the critical frequency vs. IAP for each patient

A linear regression linking $\mathrm{S}_{11}$ and IAP was proposed for each patient. Figure 5 presents the relative changes in the reflection coefficient $S_{11}$ at the critical frequency (with corresponding uncertainty (standard deviation)), vs. IAP for each patient- the differences in slope are justified by the differences in the abdominal wall compliance [14].

Given that the abdominal wall's compliance is linked to the $\mathrm{BMI}$, and it influences the slope of $\mathrm{S}_{11}$ vs. IAP, we propose that there might exist a correlation between BMI and reflectometry results. Figure 6 presents values of the slope of $\Delta S_{11}$ vs. IAP, as a function of the patients' BMI. Patient $E$ was not included in this graph since the antenna was placed in another section of the abdominal wall (see Sect. 3.1) [4].

\section{Discussion}

Reflectometry results over five laparoscopic surgery patients clearly show an inverse correlation between IAP and the reflection coefficient; these results are supported by theoretical considerations [14]. Even though the dielectric properties of tissues are well determined and have relatively low variance $[23,24]$, different subjects usually have different widths of each abdominal layer, especially fat and skin $[14,15]$. It follows that the values of $\mathrm{S}_{11}$ are expected to vary with the BMI, as seen in this trial (Fig. 6). Thicker layers of fat screen the compression of the inner layers, thus, affecting the sensitivity of the whole measurement.

Patient $\mathrm{E}$ was operated for appendectomy and the procedure required placing the antenna on the superior-left quarter of the abdominal wall (next to the diaphragm and over the limit of the abdominal cavity), which has comparably smaller compliance. Therefore, we can argue that relative changes in the AWTh (given by increments in IAP) are smaller in the leftsuperior quarter [4], rendering a low sensitivity of the AWTh to changes of IAP. In consequence, the sensitivity of $S_{11}$ is also relatively lower, as seen in Fig. 5.

Regarding abdominal compressibility, it can be argued that no evident dependence on the patient's gender has been reported in the literature.

A better understanding of the dynamics in the changes of the AWTh (during IAH) will render a more solid correlation model between BMI, the compression of the abdominal wall, and reflectometry. Among the sources of error in the measurement, we can find ventilation and motion. Any mechanical change in the abdominal wall, where the antenna is placed, will render strong artifacts in the measurements.

It must be noted that the current development of the system is able to assess changes in IAP; however, measurement of absolute values of IAP using microwave reflectometry remains a challenge. 
Fig. 6 Slope of reflection coefficient vs. IAP as a function of BMI and body mass classification

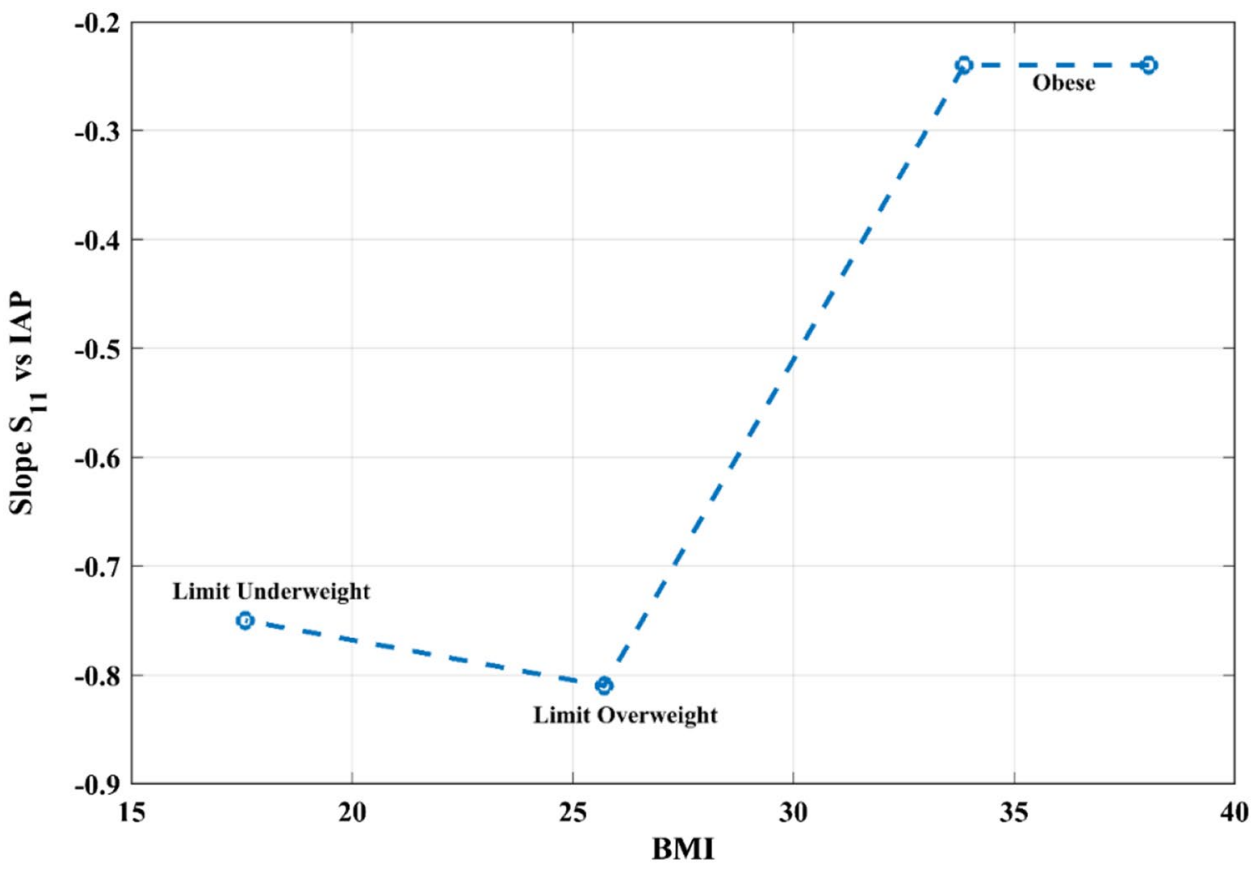

\section{Conclusions}

A microwave reflectometry system named AbdoRF was developed and clinically tried for proof-of-concept on five patients during laparoscopic surgery, which aims to estimate changes in IAP. Dependence of the reflection coefficient on the BMI is hypothesized.

Further research is still needed to optimize the sensitivity of the system; this includes optimal placement of the antenna, and redesign of the antenna itself to get larger variations of $S_{11}$ for changes in IAP.

This work presents results for IAP up to $14 \mathrm{mmHg}$, due to the standards for laparoscopic surgery. Future studies will address the study of cases for which IAP is larger as in the cases of ACS.

The applicability of this system and technique relies on a better understanding of the dynamics of the abdominal wall compressibility under the mechanical stress of IAP. Further research towards a numerical model of the AWTh dependence on IAP is needed to address the challenge of measuring absolute values of IAP by means of microwave reflectometry. Based on such a numerical model, the use of microwave reflectometry could be developed into a novel easy-to-use technique for performing a continuous noninvasive assessment of IAP.

Acknowledgements The authors warmly thank Prof. Alberto Biestro, head of the Critical Care Unit at the Hospital de Clínicas, Associate Prof. Roberto Taruselli, and Prof. Juan Riva. This research was funded by Lev Academic Center, internal research support-Grant Number 5777-8.
Funding Funding was provided by Jerusalem College of TechnologyLev Academic Center (Grant Number 5777-8).

\section{Compliance with ethical standards}

Conflict of interest The authors declare that they have no conflict of interest.

Ethical approval The proof-of-concept clinical trial was conducted on laparoscopy patients. The trial was approved by the Ethical Committee of the Hospital de Clínicas-Universidad de la República in Uruguay, in accordance with local and international regulations (permit 0512/18).

Informed consent All patients and families were informed, voluntarily accepted, and signed the required informed consent.

\section{References}

1. De Waele JJ, De Laet I, Kirkpatrick AW, Hoste E. Intra-abdominal hypertension and abdominal compartment syndrome. Am J Kidney Dis. 2011;57(1):159-69.

2. Malbrain ML, Cheatham ML, Kirkpatrick A, Sugrue M, Parr M, De Waele J, Balogh Z, Leppäniemi A, Olvera C, Ivatury R, D’Amours S, Wendon J, Hillman K, Johansson K, Kolkman K, Wilmer A. Results from the international conference of experts on intra-abdominal hypertension and abdominal compartment syndrome. I. Definitions. Intensive Care Med. 2006;32(11):1722-32.

3. Sugrue M, Bauman A, Jones F, Bishop G, Flabouris A, Parr M, Stewart A, Hillman K, Deane SA. Clinical examination is an inaccurate predictor of intra-abdominal pressure. World J Surg. 2002;26(12):1428-31.

4. Malbrain ML, Roberts DJ, De Laet I, De Waele JJ, Sugrue M, Schachtrupp A, Duchesne J, Van Ramshorst G, De Keulenaer B, Kirkpatrick AW, Ahmadi-Noorbakhsh S, Mulier J, Ivatury R, 
Pracca F, Wise R, Pelosi P. The role of abdominal compliance, the neglected parameter in critically ill patients - a consensus review of 16. Part 1: definitions and pathophysiology. Anaesthesiol Intensive Ther. 2014;46(5):392-405.

5. Malbrain ML, De Laet I, De Waele JJ, Sugrue M, Schachtrupp A, Duchesne J, Van Ramshorst G, De Keulenaer B, Kirkpatrick AW, Ahmadi-Noorbakhsh S, Mulier J, Pelosi P, Ivatury R, Pracca F, David M, Roberts DJ. The role of abdominal compliance, the neglected parameter in critically ill patients-a consensus review of 16. Part 2: measurement techniques and management recommendations. Anaesthesiol Intensive Ther. 2014;46(5):406-32.

6. Pracca F, Biestro A, Gorrassi J, David M, Simini F, Cancela M. ABDOPRE: an external device for the reduction of intra-abdominal pressure. Preliminary clinical experience. Rev Bras Ter Intensiva. 2011;23:238-41.

7. Malbrain ML. Different techniques to measure intra-abdominal pressure (IAP): time for a critical re-appraisal. In: Applied physiology in intensive care medicine. Berlin: Springer; 2006. p. 105-119.

8. Kirkpatrick AW, Roberts DJ, De Waele J, Jaeschke R, Malbrain ML, De Keulenaer B, et al. Intra-abdominal hypertension and the abdominal compartment syndrome: updated consensus definitions and clinical practice guidelines from the World Society of the Abdominal Compartment Syndrome. Intensive Care Med. 2013;39(7):1190-206. https://doi.org/10.1007/s0013 4-013-2906-z.

9. Malbrain ML, Chiumello D, Pelosi P, Wilmer A, Brienza N, Malcangi V, Bihari D, Innes R, Cohen J, Singer P, Japiassu A. Prevalence of intra-abdominal hypertension in critically ill patients: a multicentre epidemiological study. Intensive Care Med. 2004;30(5):822-9.

10. Pracca F, Biestro A, Moraes L, Puppo CB, Calvo SM, Gorrasi J, Cancela M. Direct measurement of intra-abdominal pressure with a solid microtranducer. J Clin Monit Comput. 2007;21(3):167-70.

11. Iberti TJ, Lieber CE, Benjamin E. Determination of intra-abdominal pressure using a transurethral bladder catheter: clinical validation of the technique. Anesthesiology. 1989;70(1):47-50.

12. Cheatham ML, Safcsak K. Intraabdominal pressure: a revised method for measurement. J Am Coll Surg. 1998;186(5):594-5.

13. David M, Berkovich U, Pracca F. A numerical analysis towards the continuous non-invasive assessment of intra-abdominal pressure in critical patients based on bioimpedance and microwave reflectometry. In: 13th IEEE Biomedical Circuits and Systems Conference, 2017, p. 21-24. https://doi.org/10.1109/BIOCA S.2017.8325053.
14. David M, Raviv A, Peretz A, Berkovich U, Pracca F. Towards a continuous non-invasive assessment of intra-abdominal pressure based on bioimpedance and microwave reflectometry: a pilot run on a porcine model. Biomed Signal Process Control. 2018;44:96-100.

15. Grevious MA, Cohen M, Shah SR, Rodriguez P. Structural and functional anatomy of the abdominal wall. Clin Plast Surg. 2006;33(2):169-79.

16. Caix P. Anatomy of the abdominal wall [Article in French]. Ann Chir Plast Esthet. 1999;44(4):289-311.

17. Ger R. The clinical anatomy of the anterolateral abdominal wall musculature. Clin Anat. 2009;22(3):392-7.

18. Quinn TH, Annibali R, Dalley AF 2nd, Fitzgibbons RJ Jr. Dissection of the anterior abdominal wall and the deep inguinal region from a laparoscopic perspective. Clin Anat. 1995;8(4):245-51.

19. Simini F, Bertemes-Filho P. (Eds). Bioimpedance in biomedical applications and research. New York: Springer; 2018.

20. LH Deng, SH Karagiannoglou, WI Sakkas, JC Barbenel. Effect of geometrical factors of coplanar electrodes on biomedical measurements as exemplified by the electrical impedance measurement of saline and blood. In: Proceeding of the second joint EMBS/BMES conference, 2002, p. 1667-1668.

21. Clausen CH, Skands GE, Bertelsen CV, Svendsen WE. Coplanar electrode layout optimized for increased sensitivity for electrical impedance spectroscopy. Micromachines. 2015;6(1):110-20.

22. Yang G, Long H, Tian H, Luo S, Huang H. Bioimpedance measurement: modeling of coplanar electrodes and impedance characterization. In: 2008 2nd International Conference on Bioinformatics and Biomedical Engineering, Shanghai, 2008, p. 1248-1251.

23. Gabriel S, Lau RW, Gabriel C. The dielectric properties of biological tissues: II. Measurements in the frequency range $10 \mathrm{~Hz}$ to 20 GHz. Phys Med Biol. 1996;41(11):2251-69.

24. Gabriel C, Gabriel S. Compilation of the dielectric properties of body tissues at RF and microwave frequencies. Internet document; https://www.dtic.mil/dtic/tr/fulltext/u2/a305826.pdf (authorized mirror at https://niremf.ifac.cnr.it/docs/DIELECTRIC/home. html). Last accessed: Nov 2019.

Publisher's Note Springer Nature remains neutral with regard to jurisdictional claims in published maps and institutional affiliations. 